\title{
Orbital Express Advanced Video Guidance Sensor: Ground Testing, Flight Results and Comparisons
}

\author{
Robin M. Pinson, ${ }^{1}$ Richard T. Howard ${ }^{2}$ and Andrew F. Heaton ${ }^{3}$ \\ NASA Marshall Space Flight Center, MSFC, AL, 35812
}

\begin{abstract}
Orbital Express (OE) was a successful mission demonstrating automated rendezvous and docking. The 2007 mission consisted of two spacecraft, the Autonomous Space Transport Robotic Operations (ASTRO) and the Next Generation Serviceable Satellite (NEXTSat) that were designed to work together and test a variety of service operations in orbit. The Advanced Video Guidance Sensor, AVGS, was included as one of the primary proximity navigation sensors on board the ASTRO. The AVGS was one of four sensors that provided relative position and attitude between the two vehicles. Marshall Space Flight Center was responsible for the AVGS software and testing (especially the extensive ground testing), flight operations support, and analyzing the flight data. This paper briefly describes the historical mission, the data taken on-orbit, the ground testing that occurred, and finally comparisons between flight data and ground test data for two different flight regimes.
\end{abstract}

\section{Introduction}

$\mathrm{T}_{\mathrm{p}}$ HE Orbital Express (OE) mission launched on March 8, 2007 was a historical mission. On May 5, 2007 OE performed the first autonomous rendezvous and capture by a United States spacecraft. The OE mission consisted of a pair of spacecraft outfitted with the hardware and software necessary to demonstrate the technical feasibility of on-orbit satellite servicing. The different operations performed during the OE mission were completely automated, and when possible autonomous, and consisted of spacecraft rendezvous, spacecraft proximity operations, spacecraft docking, spacecraft free-flyer capture, fluid transfers, and Orbital Replacement Unit (ORU) transfers. One of the key technologies tested was relative navigation sensors. The Automated Rendezvous and Capture Sensor System (ARCSS) contained three Boeing built proximity operations sensors along with the Advanced Video Guidance Sensor (AVGS) and a laser range finder.

The OE mission was primarily funded by the Defense Advanced Research Projects Agency (DARPA), supplemented by funding from Boeing and NASA. NASA Marshall Space Flight Center (MSFC) provided the flight software, ground testing, and some hardware and firmware support for the AVGS. In addition, MSFC tested the entire OE relative navigation sensor system in open-loop fashion in the Flight Robotics Laboratory. During the mission, MSFC provided operations support for the AVGS and analysis of its flight data.

Two satellites were launched in a mated configuration. The Autonomous Space Transport Robotic Operations (ASTRO) spacecraft was built by Boeing and the Next Generation Serviceable Satellite (NEXTSat) was built by Ball Aerospace. ASTRO was the chase vehicle, containing ARCSS and the rendezvous and docking software. NEXTSat was the target vehicle containing the AVGS targets and the Boeing sensors' target. An on-orbit view of NEXTSat taken by a camera on ASTRO is located in Figure 1.

The AVGS is a near field proximity sensor capable of determining six degree-of-freedom (DOF) solutions, with a long heritage at MSFC. The original Video Guidance Sensor (VGS) was built in-house after extensive development and testing, also in-house. The first Engineering Development Unit was built in the mid-1990's with the first flight units soon after. The first flight experiment was on the STS-87 mission in 1997 and a second flight experiment occurred on STS-95 in 1998. The AVGS was first developed for the Demonstration of Autonomous Rendezvous Technology (DART) mission, launched on April 15, 2005. The AVGS incorporated faster electronics and better lasers than those used on the VGS, and the AVGS moved to a mono-static optics configuration ${ }^{1}$. The OE

\footnotetext{
${ }^{1}$ Aerospace Engineer, Guidance Navigation and Mission Analysis, NASA MSFC, AL 35812 / EV42, AIAA member.

${ }^{2}$ Team Leader, Advanced Vehicle Sensors Team, NASA MSFC, AL 35812 / ES62, non-member.

3 Aerospace Engineer, Guidance Navigation and Mission Analysis, NASA MSFC, AL 35812 / EV42, AIAA member.
} 


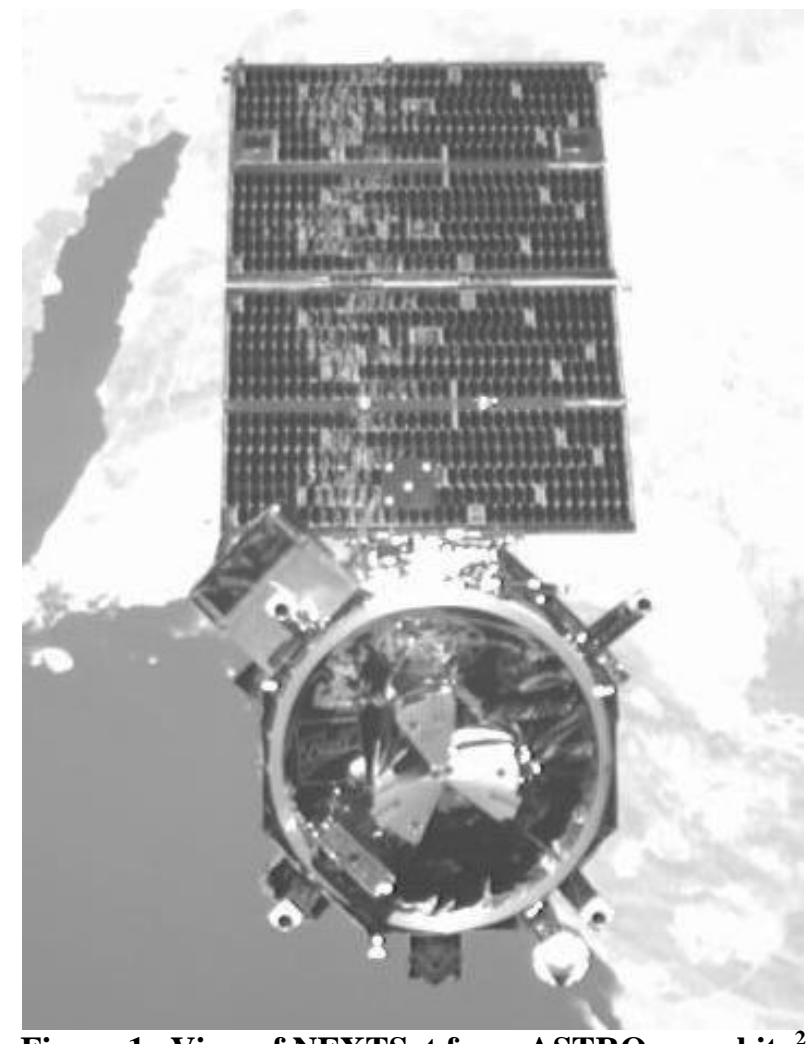

Figure 1. View of NEXTSat from ASTRO on-orbit. ${ }^{2}$

AVGS was based on the DART AVGS, except for software changes and other minor modifications. The OE mission was the first time that the full 6-DOF AVGS solution was sent to a satellite's guidance system during closed-loop operations. OE was a successful mission with the AVGS recording large amounts of data while on-orbit. The extensive ground testing prior to launch was carried out at MSFC, and it also provided large amounts of data from the AVGS. This paper contains an overview of the flight and ground testing, along with applicable comparisons between the flight and ground data.

\section{Orbital Express Flight Description}

The AVGS was a near-field proximity operations sensor integrated into ASTRO's ARCSS, which provided relative state knowledge to the ASTRO GN\&C system. The AVGS combines video, lasers, corner cubes, a frame grabber, and processing software to determine the relative state between the two vehicles. The OE mission used four smaller cornercubes for the Short Range Target (SRT), and four larger corner-cubes for the Long Range Target (LRT) located on the NEXTSat. In Figure 1, the SRT is located at the bottom center of the spacecraft, the LRT corner cubes are located approximately 45 degrees from the vertical, on the top right, top left, bottom right and bottom left portions of the spacecraft. Lasers are fired at two different wavelengths and strike the corner-cube reflectors located on NEXTSat. One laser wavelength is blocked by a filter on the corner-cubes, while the other wavelength is passed through the filter and reflected back to the sensor. During the firing of each laser frequency, the AVGS captures an image. One image thus contains all background lighting plus the corner-cube reflections, while the other image has the background lighting without the corner-cube reflections. These images are subtracted from one another and spots from the corner-cube reflectors are readily visible in the resulting image, even at long ranges and under a variety of lighting conditions. An algorithm, inverse perspective, uses this image to determine the relative range, azimuth, elevation, and attitude between the AVGS and its targets on NEXTSat. Once the relative position and attitude data has been computed, the sensor transmits the data to the navigation system, along with data on all of the spots being tracked. The sensor also sends status information as well as housekeeping data which monitors sensor temperatures, voltages, and laser output powers. The AVGS one-sigma accuracy specifications for the OE mission are located in Table 1 . As can be seen in the table, as the range between ASTRO and NEXTSat decreases, the accuracy tolerances also decrease.

Following deployment of the OE payload, initial mission operations consisted of several fluid transfer and ORU transfer operations that were conducted in the mated configuration. The spacecraft were also maneuvered relative to one another using the robotic arm on ASTRO, to check out the relative navigation sensor systems. Beginning on May 5, 2007, the spacecraft were allowed to separate relative to one another and undergo a series of Automated Rendezvous and Docking (AR\&D) missions with different characteristics and a variety of maximum separation ranges. In between

Table 1. Orbital Express AVGS Accuracy Requirements.

\begin{tabular}{|c|c|c|c|c|}
\hline $\begin{array}{l}\text { Operating } \\
\text { Range } \\
\text { (m) }\end{array}$ & $\begin{array}{l}\text { Range } \\
(\mathbf{m m})\end{array}$ & $\begin{array}{l}\text { Azimuth, } \\
\text { Elevation } \\
\text { (deg) }\end{array}$ & $\begin{array}{c}\text { Roll } \\
\text { (deg) }\end{array}$ & $\begin{array}{l}\text { Pitch, } \\
\text { Yaw } \\
\text { (deg) }\end{array}$ \\
\hline $1-3$ & \pm 12 & \pm 0.033 & \pm 0.13 & \pm 0.20 \\
\hline$>3-5$ & \pm 35 & \pm 0.033 & \pm 0.25 & \pm 0.33 \\
\hline$>5-10$ & \pm 150 & \pm 0.035 & \pm 0.45 & \pm 0.70 \\
\hline $\begin{array}{l}>10-30 \\
\text { (SRT) }\end{array}$ & \pm 1500 & \pm 0.037 & \pm 1.30 & \pm 2.0 \\
\hline $\begin{array}{l}>10-30 \\
\text { (LRT) }\end{array}$ & \pm 150 & \pm 0.027 & +0.15 & \pm 0.70 \\
\hline$>30-50$ & \pm 400 & \pm 0.030 & \pm 0.25 & \pm 1.2 \\
\hline$>50-100$ & \pm 1666 & \pm 0.033 & \pm 0.50 & \pm 2.4 \\
\hline$>100-300$ & $\pm 15,000$ & +0.035 & \pm 1.40 & \pm 7.0 \\
\hline
\end{tabular}


fluid and ORU transfers occurred. AVGS was only used in the unmated scenarios and during relative navigation check-out.

Orbital Express had six major unmated scenarios (including the end of life operations). The AVGS was used in every unmated scenario as well as in the Guidance and Relative Navigation (G\&RN) calibrations - the AT_UTIL-25 procedure - and some additional checkouts. A list of the major operations involving AVGS, along with a brief description and operation date, is located in Table 2. In order to give an idea of what the scenarios look like, plots of the AVGS range, azimuth, and elevation data from the entire Scenario 2-1 are shown in Figure 2, with the relative pitch, yaw, and roll plots shown in Figure 3. The LRT solution is in green and the SRT solution is in blue.

Table 2. Orbital Express Operations During Which AVGS Recorded Data.

\begin{tabular}{|c|c|c|}
\hline Operation & Date (UTC) & Description \\
\hline $\begin{array}{c}\text { Scenario 1-1 Berthing } \\
\text { Operation }\end{array}$ & $4 / 17 / 07$ & $\begin{array}{c}\text { Berthing of NEXTSat to ASTRO after initial } \\
\text { undocking to remove separation ring. AVGS active } \\
\text { prior to and during berthing. }\end{array}$ \\
\hline AT-UTIL-25 I & $4 / 18 / 07$ & $\begin{array}{c}\text { Performance of AT-UTIL-25, the G\&RN Calibration } \\
\text { Procedure, 44 minutes of mated AVGS data }\end{array}$ \\
\hline $\begin{array}{c}\text { Scenario 1-1A ARCSS } \\
\text { Checkout }\end{array}$ & $4 / 26 / 07$ & $\begin{array}{c}\text { The NEXTSat was moved through a series of static } \\
\text { positions on the ASTRO robotic arm to calibrate } \\
\text { ARCSS. AVGS active throughout operation. }\end{array}$ \\
\hline AT-UTIL-25 II & $5 / 4 / 07$ & $\begin{array}{c}\text { A repeat of G\&RN calibration procedure prior to first } \\
\text { fully unmated operation. 22 minutes of mated data. }\end{array}$ \\
\hline Scenario 2-1 & $5 / 5 / 07$ & $\begin{array}{c}\text { First AR\&D operation for American space program. } \\
\text { ASTRO separated to 10 meters, returned, and docked. } \\
\text { AVGS active for duration. }\end{array}$ \\
\hline Scenario 3-1, & $5 / 12 / 07-$ & $\begin{array}{c}\text { Unmated operation originally designed to be a } \\
\text { separation to 30 meters, station-keep, and return to } \\
\text { dock. Due to anomalies, range was as great as 6 km. } \\
\text { AVGS was primary sensor for final docking. Extensive } \\
\text { AVGS Acquisition data at longer ranges. The return } \\
\text { trip and docking also referred to as 3-1 Recovery. }\end{array}$ \\
\hline Sensor Stress Test & $5 / 20 / 07$ & 5 hours of static AVGS data while mated. \\
\hline Scenario 5-1 & $6 / 16 / 07$ & $\begin{array}{c}\text { Separation to 120 meters, fly-around, and return to } \\
\text { dock. AVGS active for duration. }\end{array}$ \\
\hline Scenario 7-1 & $6 / 22 / 07$ & $\begin{array}{c}\text { Separation to 3 km, station-keep, fly-around, return to } \\
\text { dock. AVGS active on departure and approach to dock. }\end{array}$ \\
\hline Scenario 8-2 & $6 / 27 / 07$ & $\begin{array}{c}\text { Separation to 7 km, station-keep, fly-around, return to } \\
\text { dock. AVGS active on departure and approach to dock. }\end{array}$ \\
\hline $\begin{array}{c}\text { Final separation of ASTRO and NEXTSat prior to } \\
\text { mission termination. AVGS active on departure. At the } \\
\text { end of EOL, ASTRO and NEXTSat were in different } \\
\text { orbits and permanently powered down. }\end{array}$ \\
\hline
\end{tabular}

Two special areas of operation for which the data was closely examined were from the Dual Track portions and the mated portions of the scenarios. During a typical mission the SRT would be visible to the AVGS from the docked configuration until slightly beyond a relative range of 30 meters. The LRT would first be tracked by the AVGS at a relative range less than 10 meters and the AVGS would continue tracking it until the LRT left the AVGS's field of view (FOV), due to ASTRO's maneuvers. This region where both the SRT and LRT gave solutions is commonly referred to as the Dual Track region. This is seen between $900-6400$ seconds in Figure 2 and Figure 3 , where the green and blue lines overlap. Most scenarios have two distinct Dual Track sections, one on departure from the NEXTSat and the other on the approach. For Scenario 2-1, the entire scenario stayed within the SRT operating range, thus it is one long Dual Track sample instead of two distinct samples.

The only true static data taken on-orbit was when the two vehicles were in mated configuration. The docking mechanism used for OE had a very tight tolerance that was essentially below the threshold of the AVGS to detect, and thus made an excellent source of "truth data." This was the only real source of truth data available on-orbit. The majority of the mated data samples were three minutes or less in length occurring prior to de-mate or immediately 
after the vehicles were docked. This can be faintly seen at the beginning and end of the range plot in Figure 2. The three longer mated data samples occurred during the sensor stress test and the two AT_UTIL-25 operations. The stress test was the longest mated sample taken throughout the project at 5.2 hours.

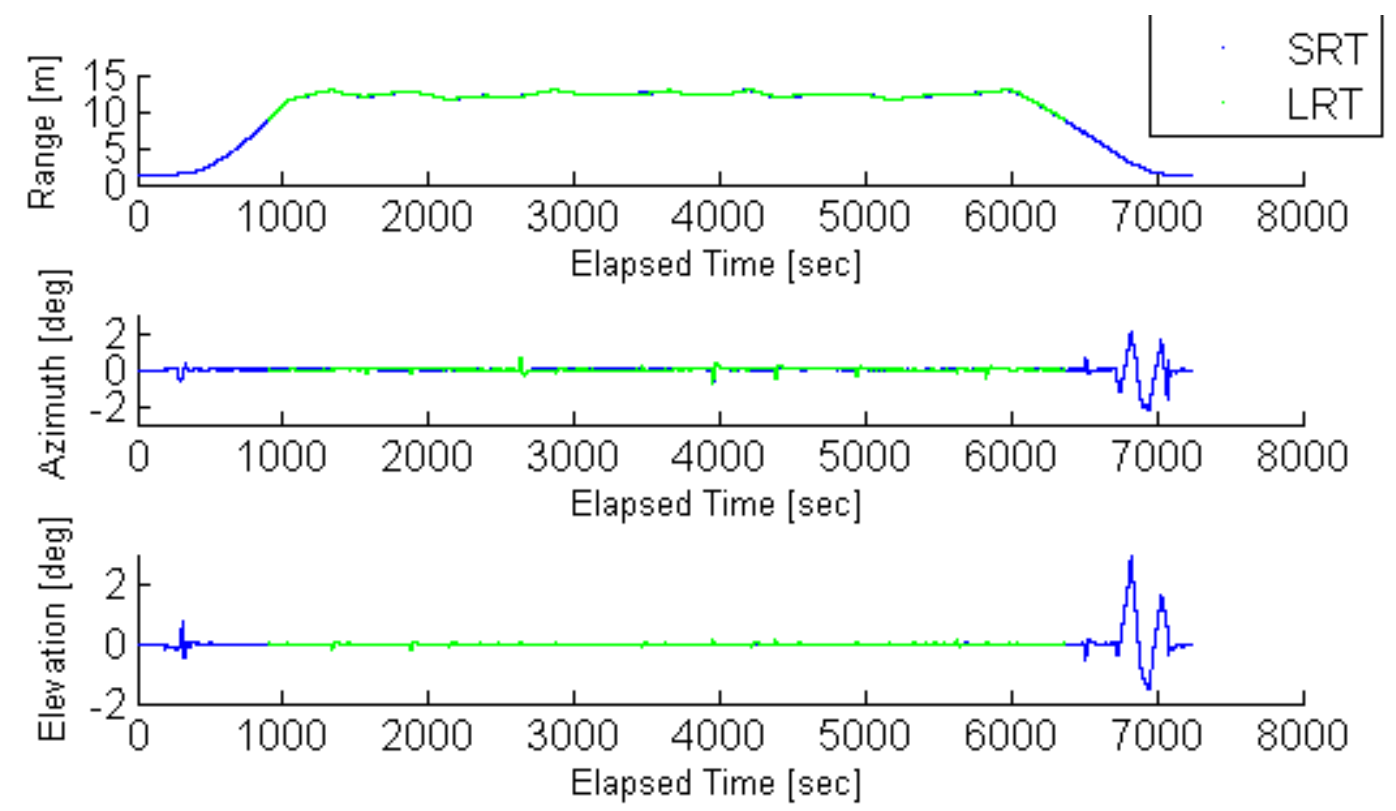

Figure 2. Relative Range, Azimuth, and Elevation from OE Scenario 2-1. SRT blue, LRT green

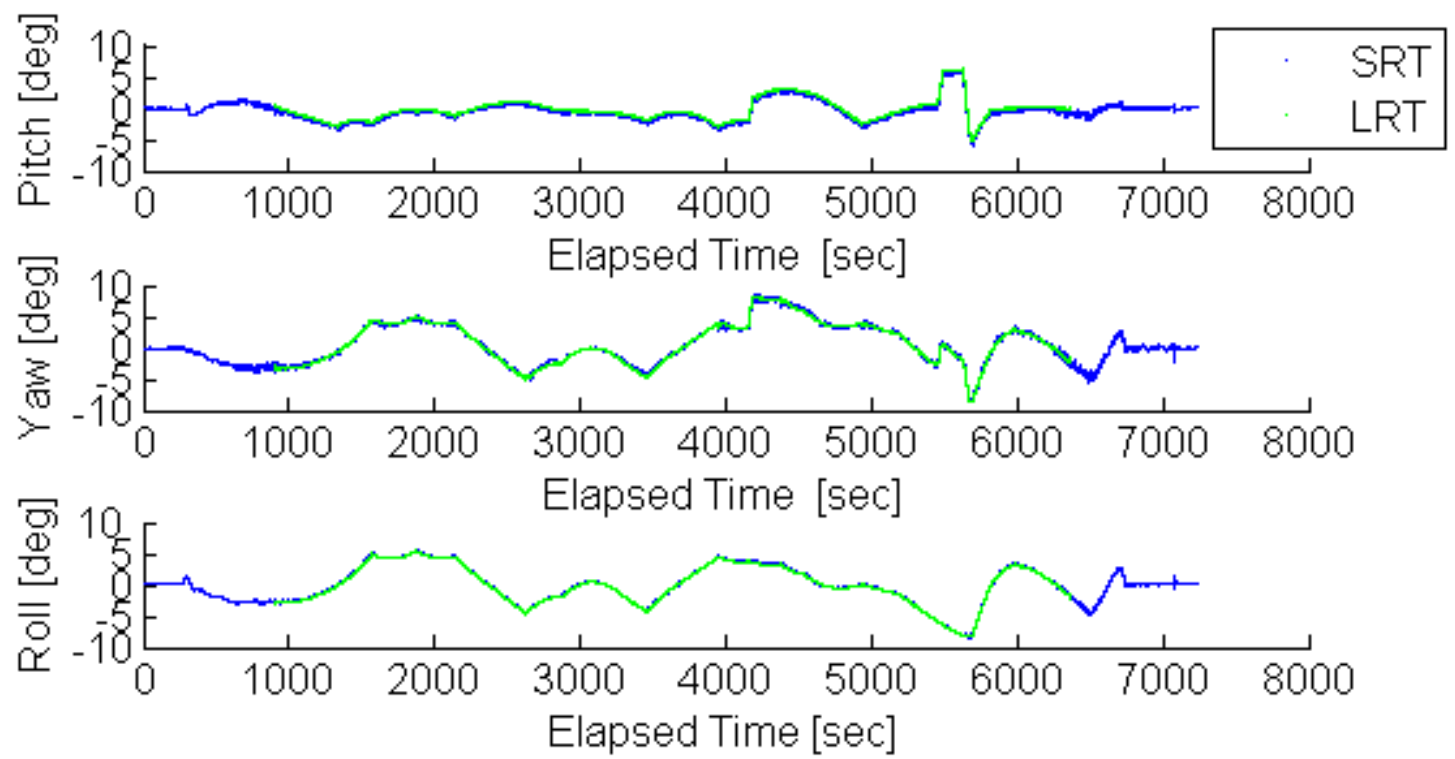

Figure 3. Relative Pitch, Yaw, and Roll from OE Scenario 2-1. SRT blue, LRT green.

\section{Ground Test Demonstration Description}

One of the lessons learned from this project was the extreme importance of extensive ground testing. It could safely be said that every new test uncovered some previously unknown problem with the sensor. The testing, followed by the correction of the discovered problems, took considerable time, but the time was well spent as 
evidenced by the excellent performance of the sensor on-orbit. Much of the testing was similar to that performed for the DART AVGS ${ }^{3}$, but for OE all of the testing was carried out in one building at MSFC.

One of the main ground tests was the Optical Characterization Testing (OCT) of the sensor with both the SRT and the LRT over a variety of ranges, attitudes, and locations in the field of view. For the OE AVGS, the OCT testing consisted of creating the best combination of values for the sensor integration time (i.e. the exposure time for each picture), threshold, and laser power for use at each range. In AVGS operations, the threshold is used to prevent dim spots (which are usually noise-based data) from interfering with the target recognition and processing. The laser power can be varied from $10 \%$ to $100 \%$ power, and the integration time can vary from 6 uS to several thousand uS. Based on prior work with video sensors, a laser power profile was chosen a priori, as was the threshold level, so the only variable determined for each operational range during OCT was the sensor's integration time. At each test range, the AVGS was run through a series of integration time values while viewing the SRT in the center of the FOV. The value that caused the target image to just reach saturation, when the target image had intensity values of 255, was the value chosen for the integration time at that range. Prior testing had determined that if the target was near saturation in the center of the FOV, then the target would still be visible in the corners of the FOV, despite the lowered laser intensity. The relative ranges used for OCT were 1, 2, 3, 5, 10, 15, 25, 30, 35, 50, 75, and 100 meters. Then the sensor was rotated to ensure that even in the corners of the FOV that the chosen parameters would effectively allow the target to be tracked. The corner location was at $(6,6)$ degrees in the FOV. Finally, the sensor was maneuvered horizontally (side-to-side) and vertically (up-and-down) to allow the target to be scanned across the entire FOV. This ensures that the integration time, threshold, and laser power settings were correct for the entire area specified in the performance requirements. The FOV plots show each point at which the AVGS was tracking its target. The FOV plot for a relative range of three meters is located in Figure 4. Within the 25 to 30 meter range, there were some problems with the flight unit's performance. There were unanticipated defects in the optics that led to dropouts in the FOV test (see Figure 5) and they had to be compensated for by extra testing and careful choice of the parameters. For details on the corrections see Ref. 4.

3 meter FOV plot

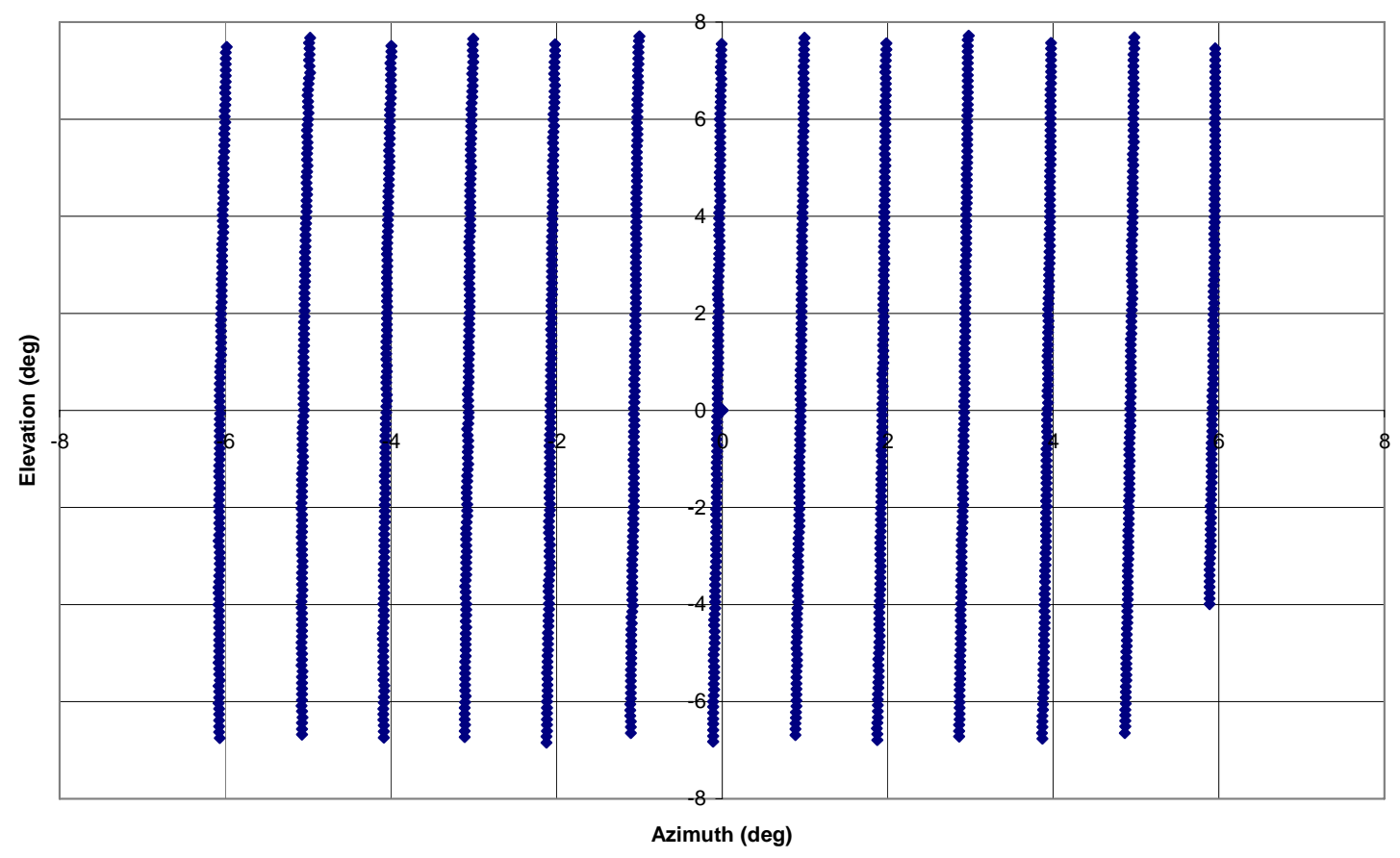

Figure 4. AVGS Field-of-View with the Short-Range Target at 3 meters of Range. Points Represent Successfully Tracked FOV Locations. 


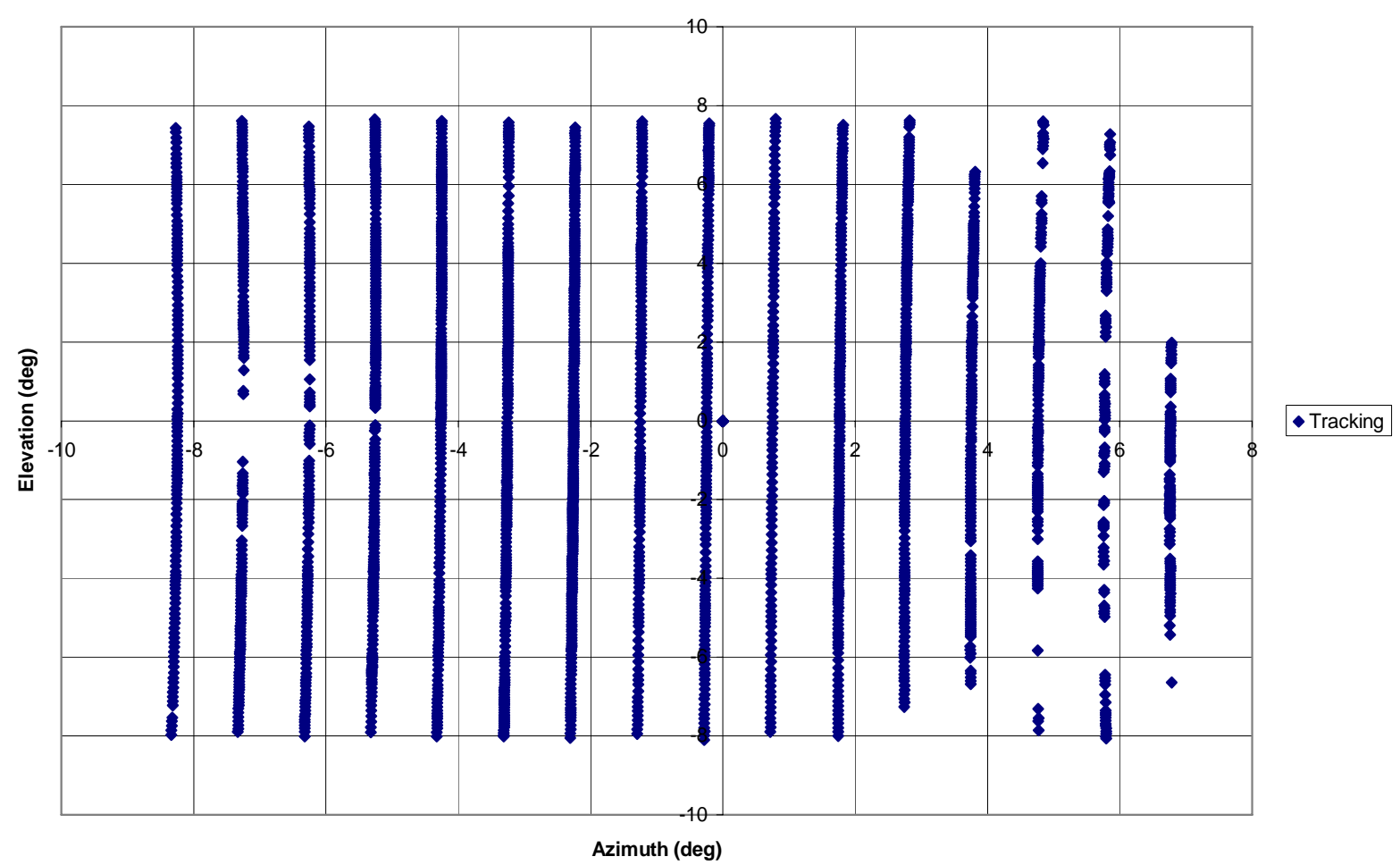

Figure 5. Field-of-View Plot of the AVGS SRT at 30 meters of Range - Notice Dropouts at Right and Center Left.

Once the values for each test range had been determined, the information was programmed into the initial load (I-Load) of the software. The I-Load was a small set of parameters that defined settings that might change during the course of the project. As an ASCII file, the I-Load could be easily updated as necessary, even when the sensor was on-orbit. After the I-Load had been created, then the OCT Confidence test was carried out. The OCT Confidence test was a short series of tests performed at several different ranges $(1.2,1.8,3,5,9,13,18,20,28,39,56,64,75$, and 88 meters.) The OCT Confidence test was designed to ensure that the values chosen during OCT were sufficient to allow the AVGS to meet its requirements throughout the entire volume of performance space (every range, every target angle, and every position in the FOV.) Obviously, exhaustive testing was impossible as that would be an infinite test space, so a subset of ranges was chosen. The ranges chosen were generally between those used in the OCT testing, since OCT ensured that the parameters were correct for the ranges already tested. The OCT Confidence also tested a few ranges that repeated the previous OCT ranges for comparison purposes. The subset of tests consisted of FOV scans of the target at nominal and maximum tilt angles. Figure 6 contains the FOV scan for both the SRT and the LRT at 13 meters range. It was reasoned that if the sensor could track the target at its brightest and dimmest angles in the FOV, it would also track at in-between values.

The OCT was performed at a maximum relative range of 100 meters, despite the project requirement to operate up to 200 meters. This restriction was due to a combination of laboratory test limits and available time for testing in additional facilities and environments. Once the sensor was on-orbit, the test limitation of 100 meters led to the AVGS only successfully tracking out to 150 meters. The parameters for relative ranges greater than 100 meters were extrapolated from previous test data and prior sensor knowledge. The lack of performance past 150 meters on-orbit is directly tied to lack of ground testing, and could easily have been achieved with the proper tests.

Another performance parameter that the sensor was required to meet was the Total Bearing Angular Velocity (TBAV). TBAV was the requirement related to how fast the target could move across the AVGS's FOV and still be tracked. It was a single rate that could result from a combination of sensor translation, sensor rotation, target translation, and target rotation. This rate was tested by rotating the sensor at ever-increasing rates with the target starting outside the FOV, then AVGS scanned one time across the FOV, ending up on the opposite side outside the 


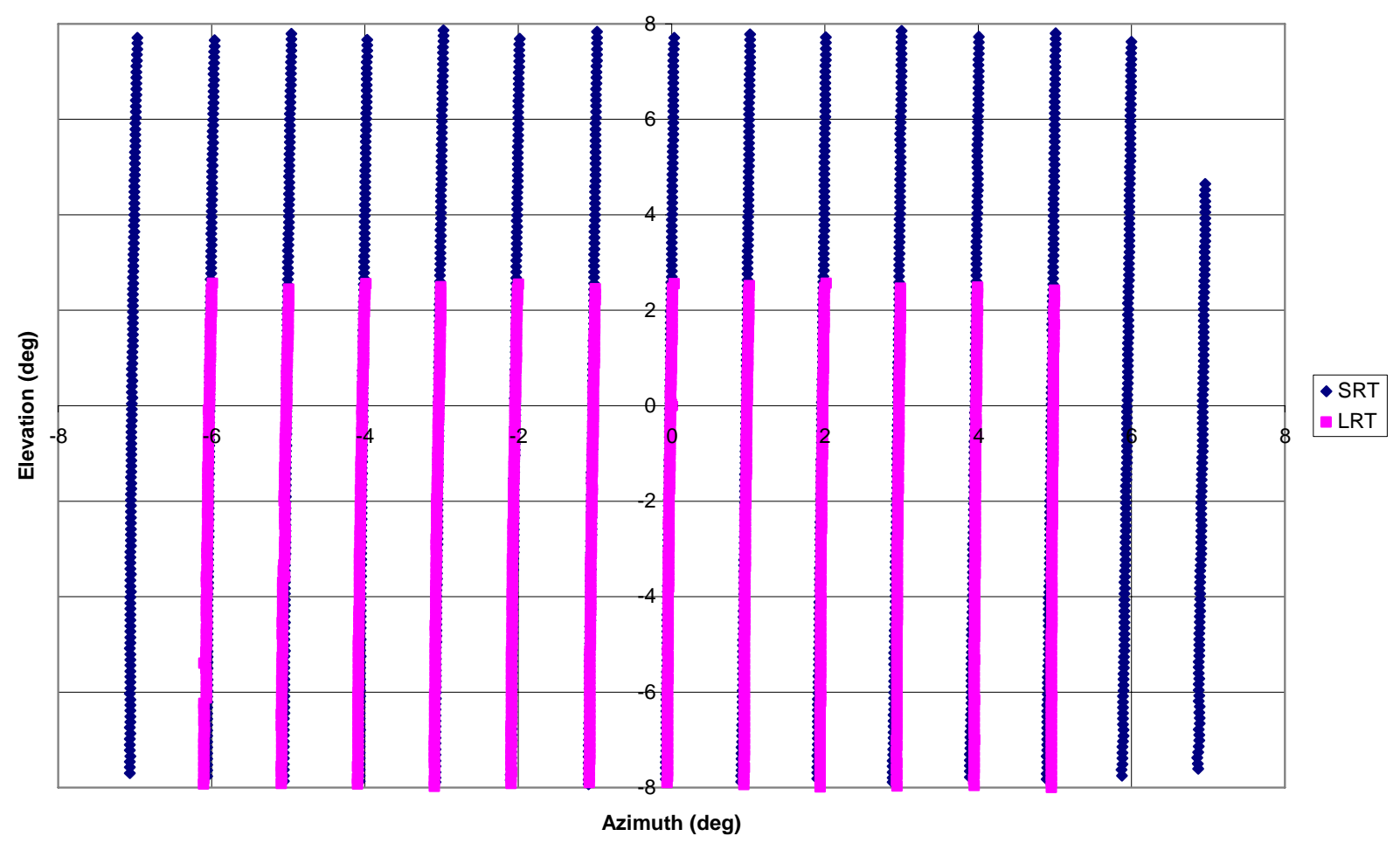

Figure 6. Field-of-View Plot of the AVGS for SRT and LRT at 13 meters of Range.

FOV. The rates tested went up to and then beyond the TBAV requirement. The AVGS could track past its required TBAV rate of 2.0 degrees per second, thus showing the sensor was within specification for this requirement.

When the flight unit was shipped to MSFC it had some known problems with the electronics. The problems were compensated for by modifying the Field Programmable Gate Array (FPGA) code (also known as firmware), but the compensation was not sufficient across the full range of operating temperatures, which was discovered once the AVGS was put in a thermal vacuum chamber. The unit then required the replacement of the image processing electronics card, so vibration and thermal vacuum testing had to be repeated. Before, during, and after each type of thermal testing, the AVGS was tested by running through its different modes of operation and by checking the boresight alignment. The boresight alignment of the sensor relative to its baseplate would directly affect the relative positions of the spacecraft during proximity operations, so it was a critical parameter. Since the boresight was fixed once the sensor was assembled, any shift in the boresight would indicate a hardware problem.

The final ground test performed was the System Test, in which the target and sensor positions were carefully measured, and then the target was moved specified amounts while the sensor tracked it. During this and other phases of testing, the Boeing-MSFC team had recourse to excellent test equipment that helped achieve a highly accurate source of truth data for the tests. The state of the art Newmark and Leica equipment helped greatly advance the proximity sensor truth data measurements. The truth data was always critical, especially for the System Test, which was the final validation of sensor accuracy prior to launch. The highly accurate truth data allowed the team to tune the sensor parameters in the I-Load following the System Test to make the sensor more accurate, and to validate this adjustment using the same set of test data. The System Test ran through a large list of commanded attitudes for each relative range tested.

\section{Mated Data Comparison}

One of the regimes for which comparison between ground data and flight data was possible was the mated data. On-orbit this occurred when the vehicles were rigidly docked. Fifteen samples were taken on-orbit, the majority from when the sensor was turned on prior to the de-mate, or the AVGS remained powered on after the final docking. 
Three additional longer samples were taken during operations when the vehicles did not undock. On-orbit the mated configuration corresponds to a relative range of 1.22 meters. The ground test data in this comparison came from static cases when the target and sensor relative range was between 1.2 and 1.8 meters. There was one case at a 3 meter range that was also included. Ten cases were chosen from the ground testing to compare with the fifteen flight cases. The samples from the System Test correspond to when the relative attitude between the sensor and target was commanded to zero. All data taken on-orbit and on the ground was static with no physical change in the relative target and sensor configurations; which allowed for the best comparison.

Figure 7 contains the azimuth versus time and elevation versus time plots for one of the longest ground tests, OCT Confidence 1.2 meters, compared to a similar sample length on-orbit. In this case, the mated data was taken after completion of Scenario 7-1, prior to powering down the sensor. The azimuth plot shows the similarities in the frequency of the noise between the two samples. Due to the set-up on the ground there were biases, that appeared in the test data that differed from the biases on-orbit. Extraneous biases were removed via an ILoad update prior to launch, decreasing the magnitude of the biases that were seen on-orbit. Also, the ground test did not have a rigid docking

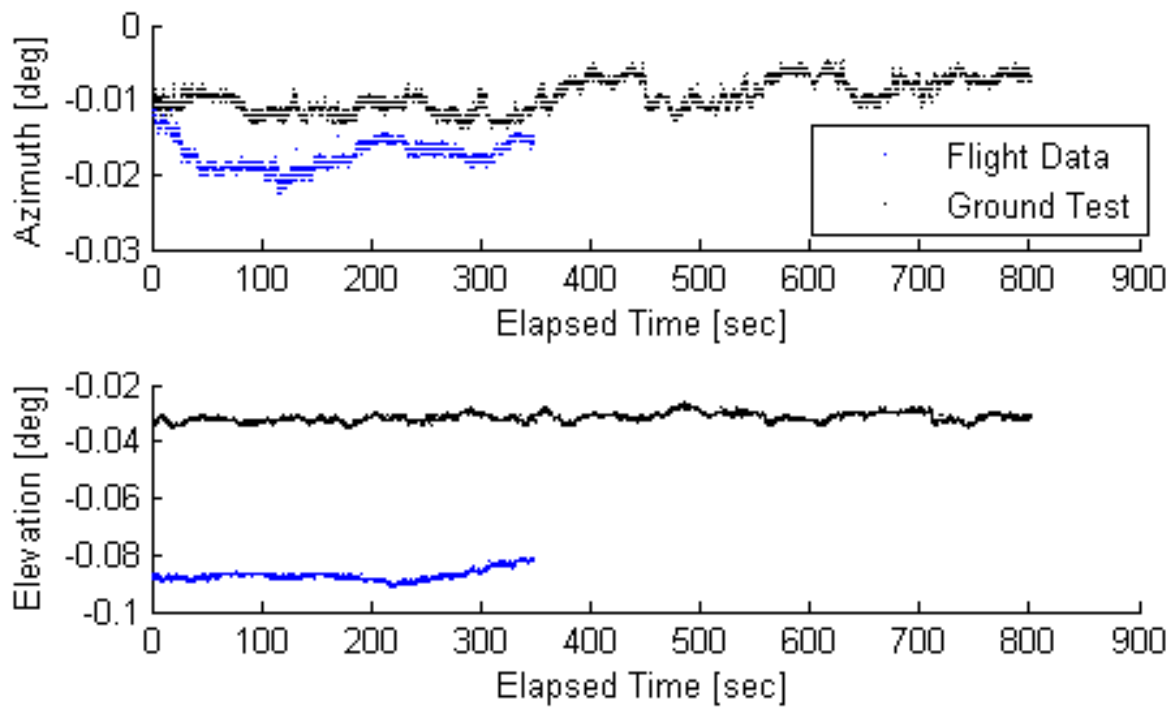

Figure 7. Comparison of Flight Data from Scenario7-1 post (blue) and Ground Test OCT Confidence $1.2 \mathrm{~m}$ (black).

clamp forcing the sensor and target in a strict repeatable configuration for every test.

Standard deviation is the best measurement used to compare the different data samples. Biases could easily occur in the ground testing when target fixtures were moved or not aligned precisely prior to the start of the test. Some of the ground test data was taken earlier in the program before biases were investigated. Therefore, comparing the means will not lead to an effective comparison between the flight and ground test data.

The accuracy specifications that apply to the mated configuration correspond to the first row (1 to 3 meter range) in Table 1. These specifications refer to the combination of noise and bias when mated. Figure 8 compares the standard deviations of the range, azimuth, and elevation between the different mated flight and ground test samples. In Figure 8, the division between the flight data and the ground test data is the gap between the Sensor Stress Test and the OCT Confidence Test. Everything to the left is the flight data and to the right are comparable ground test samples. The largest standard deviation from these samples for azimuth and elevation is less than 0.005 deg which is well under the $0.033 \mathrm{deg}$ limit. The largest range standard deviation is less than 0.0025 meters, again well under the 0.012 meter accuracy requirement. (This maximum magnitude only occurred at the range of 3 meters; other standard deviations were much smaller at closer ranges.) This leaves room for small biases without violating the accuracy specification. Small biases did appear in the flight data, but the project deemed it unnecessary to remove them via a new I-Load. Overall the statistics for the flight data and ground data appear very similar. There are no major differences in the standard deviation magnitudes or variations between similar tests. 


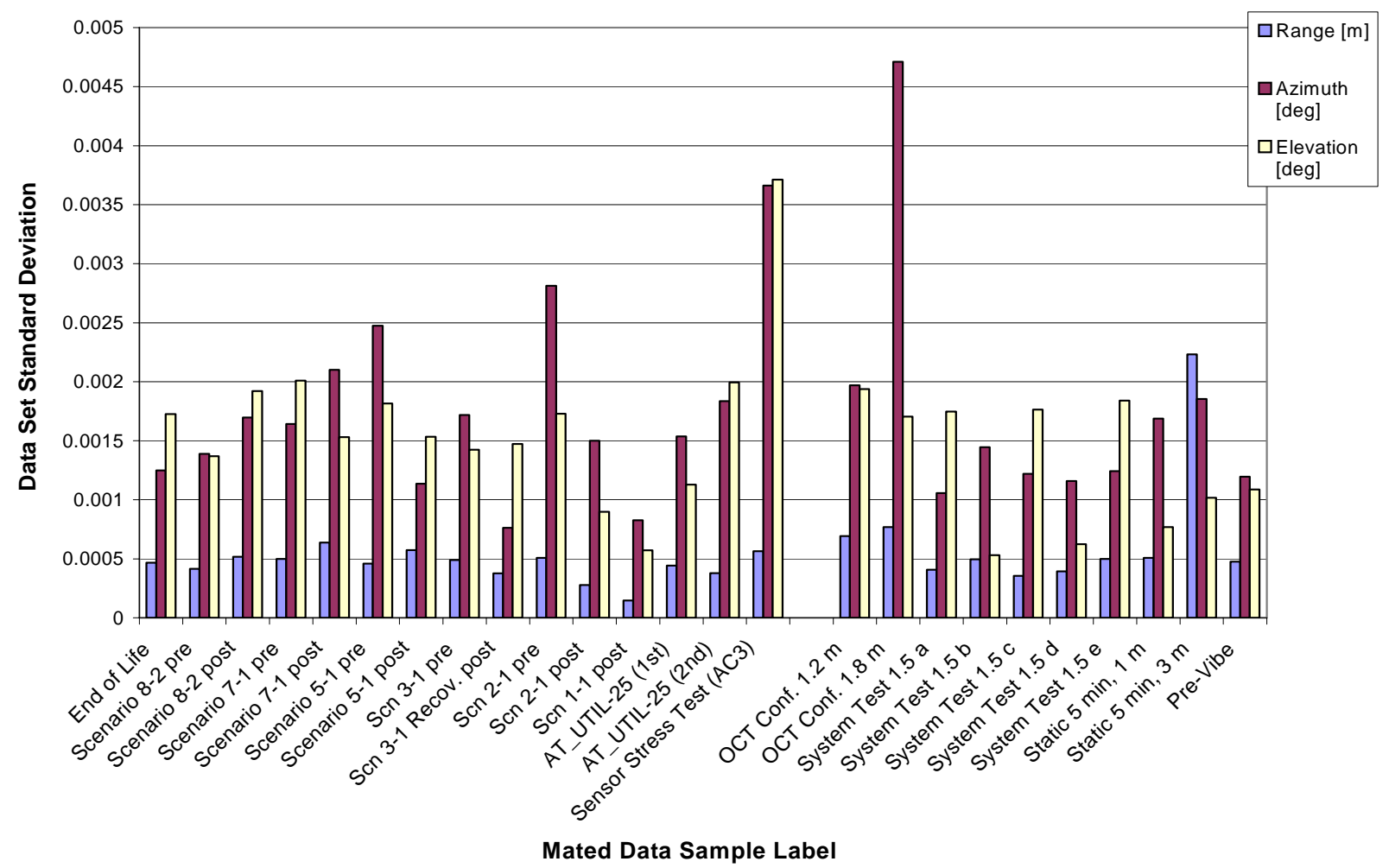

Figure 8. Comparison between Flight Data (left 15) and Ground Test Data (right 10) Range, Azimuth, and Elevation Standard Deviation.

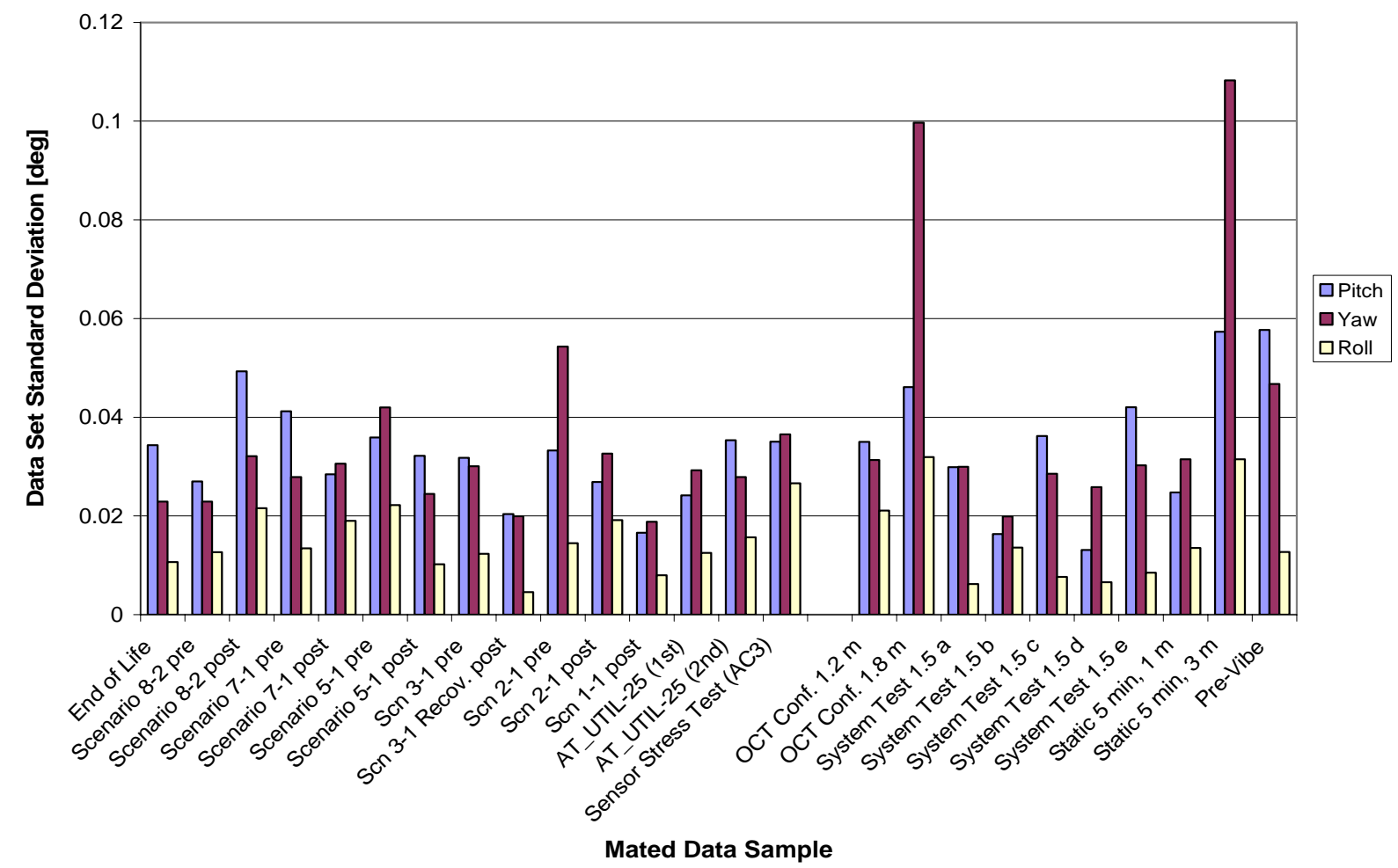

Figure 9. Comparison between Flight Data (left 15) and Ground Test Data (right 10) Pitch, Yaw, and Roll Standard Deviation. 
Figure 9 is similar to Figure 8, in that they both examine the same cases, though Figure 9 focuses on the attitude (pitch, yaw, and roll). The largest pitch or yaw standard deviation is less than 0.12 deg, which is significantly under the 0.20 deg accuracy specification. The largest roll standard deviation in the data is less than 0.04 deg, which is less than one third of the 0.13 deg requirement. As with the range, azimuth, and elevation, there are no large distinctions between the flight data and the ground test data. Twice the yaw for the ground test data was larger than the flight trends. This just emphasizes that if the sensor performed within its specification on the ground, it was likely to do as well if not better in space. The great correlations between the ground test data and the flight data lend credibility to the ground tests accurately predicting the AVGS performance envelope for the mated configuration on-orbit. Close in, almost docked, is one of the critical regions of operation for a proximity sensor. The mated data on-orbit and from the ground tests show high repeatability and reliability for the AVGS in the docking corridor.

\section{Dual Track Comparison}

Another way to examine the AVGS data is to compare the LRT solution to the SRT solution for the exact same instance of time, during the Dual Track regimes. During the Dual Track region, the AVGS is measuring the relative position and attitude of NEXTSat by tracking both the SRT and the LRT. At the same point in time, the two solutions should be identical, since the spacecraft cannot physically change its attitude and position between the targets. For these comparisons, the SRT solution was subtracted from the LRT solution.

Seven operations on-orbit had Dual Track samples: Scenario 2-1, Scenario 3-1 (counted as two samples), Scenario 5-1, Scenario 7-1, Scenario 8-2 and the End-of-Life operation. For Scenarios 5-1, 7-1 and 8-2, there were two distinct periods of time when the sensor was in Dual Track, which were combined into one sample per scenario. Since Scenario 3-1 and Scenario 3-1 Recovery occurred eight days apart they were treated as separate samples. There were sixteen static data samples from the ground test found for the comparison. The System Test samples were from tests when the relative attitude between target and sensor was commanded to zero degrees. Most of the static samples were taken around a relative range of 11 meters or 28 meters. Unfortunately the ground-test samples' durations were much shorter than the on-orbit samples. Half of the ground test samples were under 1.5 minutes, with

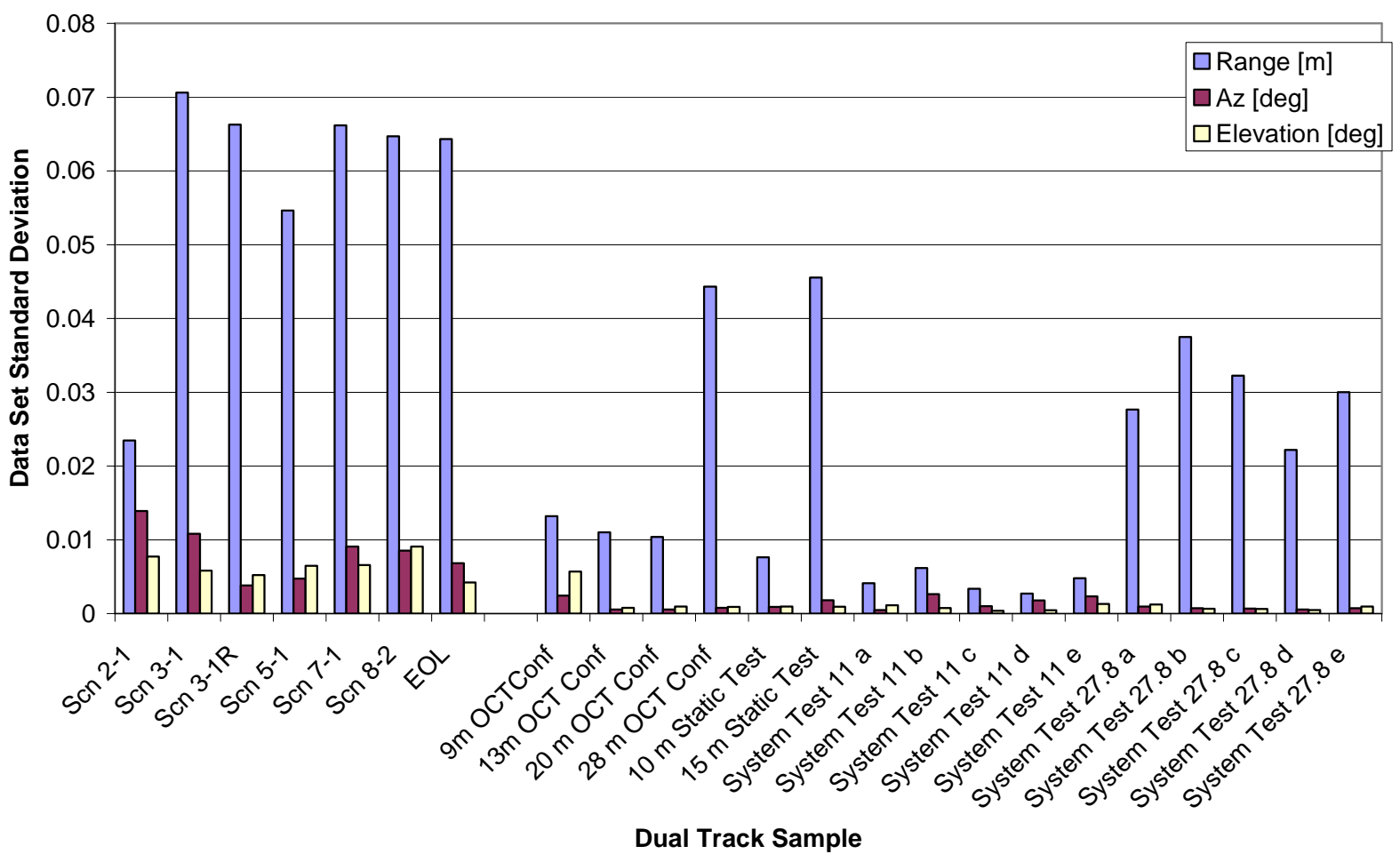

Figure 10. Comparison between Flight Data (left 7) and Ground Test Data (right 16) Range, Azimuth, and Elevation Standard Deviation. 
all the samples being less than 6.5 minutes in duration. The shortest on-orbit sample was over 13.5 minutes. The main difference between the flight data and the ground test data was that the ground test was static at a predetermined location and attitude, while the flight data was dynamic, traveling between $10 \mathrm{~m}$ and $30 \mathrm{~m}$ (or $30 \mathrm{~m}$ to $10 \mathrm{~m}$ ) relative range.

The standard deviation of the range, azimuth, and elevation from the twenty-three data samples is shown in Figure 10. All samples to the left of and including the EOL are flight data, with everything to the right of and including $9 \mathrm{~m}$ OCT Conf are ground test samples. Accuracy requirements located in the third row through the sixth row of Table 1 correspond to the Dual Track regime, with the tightest accuracy specification corresponding to the fifth row. The tightest accuracy requirements correspond to the $10-30$ meters LRT measurement specification and will be used as the Dual Track accuracy requirements. The largest standard deviation of the relative range data was under half of the accuracy requirement of 0.15 meters. The azimuth and elevation standard deviations were well under 0.01 degrees compared to the accuracy requirement of 0.027 degrees. There is a more noticeable spread between the flight data and the ground test data for the Dual Track regime. There were two major differences between the data taken prior to flight and the flight data. During the flight scenarios, ASTRO was constantly in motion relative to the NEXTSat. This would cause more variation and noise, than data taken with a fixed sensor and target location. Also, the sample lengths have an affect on the comparisons. Still, the ground test was a good check on the accuracy requirements for different ranges. Overall the range, azimuth, and elevation standard deviations were much larger at the 27.8 meter range than the 11 meter range (ground test). Every test remained well under the accuracy specifications.

Figure 11 shows the standard deviations for the pitch, yaw, and roll for the twenty-three cases under comparison. These are the same data samples as in Figure 10. All the roll standard deviations were less than or equal to the 0.15 degree accuracy requirement. Pitch and yaw standard deviation were all under 0.4 deg far less than the 0.70 degree requirement. The comparisons between the ground and flight did not have as large a difference between the flight and ground data for the attitude as for the position.

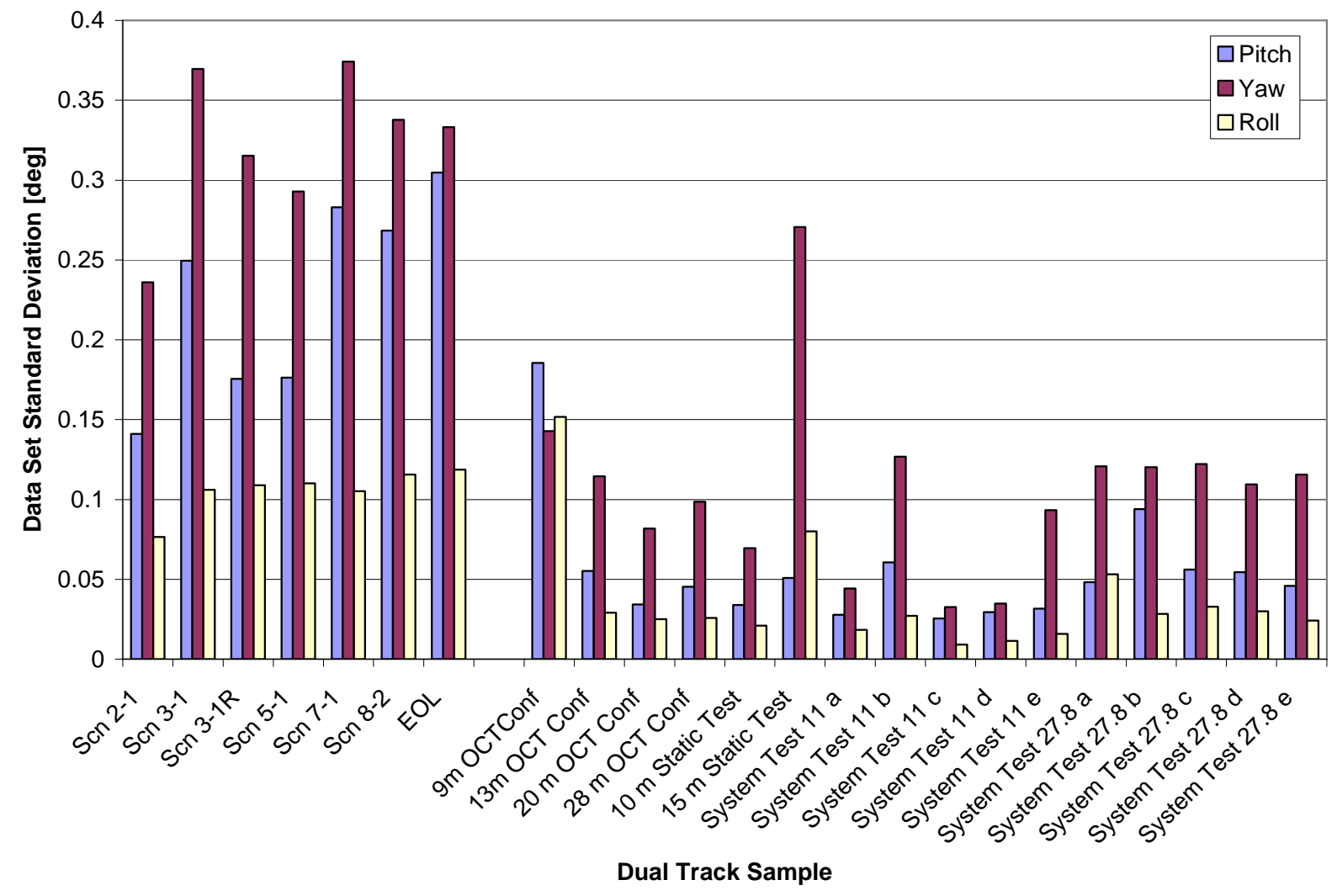

Figure 11. Comparison between Flight Data (left 7) and Ground Test Data (right 16) Pitch, Yaw, and Roll Standard Deviation. 


\section{Conclusion}

The AVGS performed admirably during the Orbital Express mission. The sensor provided crucial relative position and attitude data during every entry into the approach corridor. In addition, the ground data and the flight data were very consistent in the areas that could be accurately compared. This comparison lent credibility to the functionality of the ground test. While there is no substitute for flight data, the ground test flushed out several issues and allowed the sensor's characteristics to be understood. Since the comparisons between the ground test data and the flight data were good, future projects will have greater confidence in the fact that sufficient ground testing produces results comparable to space. Based on the excellent results of both the ground testing and the flight, the AVGS sensor technology is ready for further applications

\section{Acknowledgments}

The authors thank Lt. Col. Fred Kennedy of DARPA for his encouragement, support and leadership of the entire program; Bob Friend of Boeing for his help throughout the testing and flight; and all U.S. taxpayers for the funds necessary to carry out this innovative work.

\section{References}

${ }^{1}$ Howard, R. T., and Bryan, T. C., "DART AVGS Flight Results,” Sensors and Systems for Space Applications, edited by R. T. Howard and R. D. Richards, SPIE Conference Proceedings Vol. 6555, Bellingham, WA, 2007, pp. 1-10.

${ }^{2}$ Defense Advanced Research Projects Agency (DARPA), “Orbital Express On-Orbit Mission Updates” (2007), http://www.darpa.mil/orbitalexpress/mission_updates.html, accessed July 11, 2008.

${ }^{3}$ Howard, R. T., Johnston, A. S., Bryan, T. C., and Book, M. L., “Advanced Video Guidance Sensor (AVGS) development testing,” Spaceborne Sensors, edited by R. D. Habbit, Jr. and P. Tchoryk, Jr., SPIE Conference Proceedings Vol. 5418, Bellingham, WA, 2004, pp. 50-60.

${ }^{4}$ LeCroy, J. E., Hallmark, D. S., and Howard, R. T., "Effects of Optical Artifacts in a Laser-Based Spacecraft Navigation Sensor," in Sensors and Systems for Space Applications, edited by R. T. Howard and R. D. Richards, SPIE Conference Proceedings Vol. 6555, Bellingham, WA, 2007, pp. 1-11. 